\title{
APLICACIÓN DE UN INSTRUMENTO DE EVALUA- CIÓN EN OBJETOS DE APRENDIZAJE DEL CAMPUS VIRTUAL DE LA FRRe
}

Mgter. Liliana Cuenca Pletsch ${ }^{1}$; Ing. Valeria Sandobal Verón ${ }^{2}$; Ing. María Alejandra $\operatorname{Cernadas}^{3}(*)$

\section{Resumen}

A continuación se presenta el resultado de la evaluación de objetos de aprendizaje utilizando el instrumento propuesto en el marco del proyecto "Modelización de un repositorio de objetos de aprendizaje para la gestión del conocimiento para la UTN-FRRe". El mismo se diseñó teniendo en cuenta el modelo de Objeto de Aprendizaje (OA) y la taxonomía elegida para el proyecto, basados en el análisis y propuesta de mejora de herramientas existentes. La propuesta de evaluación considera aspectos pedagógicos, didácticos y tecnológicos.

Palabras Clave: objetos de aprendizaje, herramienta de evaluación $O A$

\section{INTRODUCCIÓN}

Los estándares para el desarrollo del e-learning marcan la pauta para crear sistemas que integren aplicaciones para los procesos de enseñanza y aprendizaje en línea, en las que los contenidos puedan ser reutilizados y compartidos, entre personas y entre sistemas. A estos se los denomina Objetos de Aprendizaje (OA), los que forman bibliotecas de aplicaciones para el ámbito educativo, llamados Repositorios de Objetos de Aprendizaje (ROA) [1].

García Aretio [2] reflexiona, respecto de la información disponible en Internet, “... Estos datos debidamente combinados producen unidades de información, pero combinados de otra manera o con otros datos pueden llegar a generar unidades diferentes. El autor plantea la reutilización de unidades de información como forma de rentabilizar la conjunción de datos que adecuadamente combinados producen información.

El Comité de Estandarización de Tecnología Educativa de la IEEE establece que los objetos de aprendizaje son "una entidad, digital o no digital, que puede ser utilizada, reu-

(*) Universidad Tecnológica Nacional, Facultad Regional Resistencia -French 414 - Resistencia (3500) - Chaco

1.Prof.Titular.cplr@frre.utn.edu.ar-0362-154563171

2. Jefe de Trabajos Prácticos. valeriasandobal@hormail.com - 0362-154 664580

3. Jefe de Trabajos Prácticos.ma_cernadas@hotmail.com - 0362-154 302755 
tilizada y referenciada durante el aprendizaje apoyado con tecnología”. Mason, Weller y Pegler [3] los definen como "una pieza digital de material de aprendizaje que direcciona a un tema claramente identificable o salida de aprendizaje y que tiene el potencial de ser reutilizado en diferentes contextos". Según Wiley [4] los objetos de aprendizaje son los elementos de un nuevo tipo de instrucción basada en el computador y fundamentada en el paradigma computacional de orientación a objetos.

Según Lowerison, Gallart y Boyd [5] los beneficios que los OA pueden tener en un contexto educativo son: flexibilidad, ya que el mismo recurso puede utilizarse en distintos contextos; administración del contenido, mediante utilización de metadatos que permiten su control; adaptabilidad, facilita al diseñador poder seleccionar y componer recursos según la aplicación; y código abierto, elimina los problemas de incompatibilidad entre plataformas.

Para poder determinar si un OA cumple con el modelo, de acuerdo a una definición funcional, se desarrollaron instrumentos para evaluar atributos pedagógicos y técnicos, entre los que se mencionan: calidad de los contenidos, potencialidad como herramienta de enseñanza, adecuación, feedback, motivación, diseño y presentación, usabilidad, accesibilidad, reusabilidad, cumplimiento de estándares.

En la Fig.1 se presenta un modelo, propuesto por Alvarez Rodriguez y Muñoz Arteaga [6], que facilita la integración de OA y se ajusta al estilo de creación de estos recursos en la Facultad Regional Resistencia (FRRe) de la Universidad Tecnológica Nacional (UTN).

\section{Teoría}

-Información que puede ser texto, imágenes, etc

- Conceptos sobre el tema abordado en el OA.

- Le permite al alumno obtener una experiencia abstracta

\section{Experimentación}

- Contiene animaciones, simulaciones, etc.

- Permite al alumno experimentar y reflexionar sobre el tema abordado en tería.

Obtiene una experiencia directa y concreta del tema.

\section{Evaluación}

- Evaluar el conocimiento adquirido por el alumno

-También podría utilizarse para que el alumno evalúe el grado de utilidad del OA

\section{Información relacionada}

- Referencias a otros OA de temas relacionados, que el alumno puede consultar

Fig.1. Modelo de OA 
Con respecto a la taxonomía se presenta, en la fig.2, una que clasifica los OA según los recursos, con fines completamente pedagógicos [7].
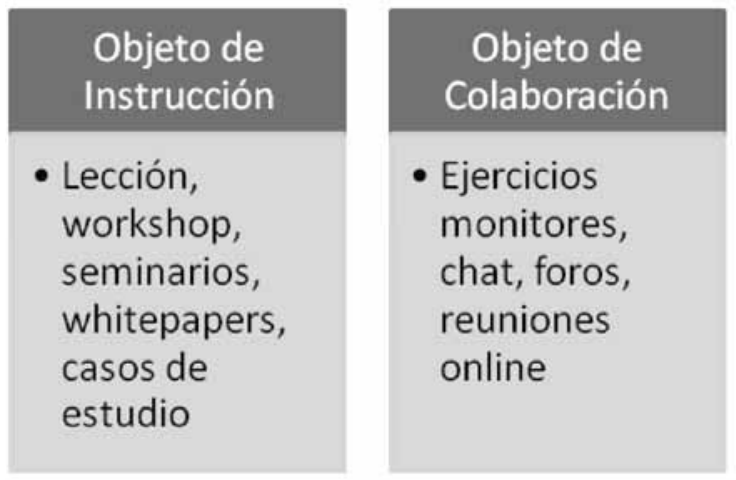

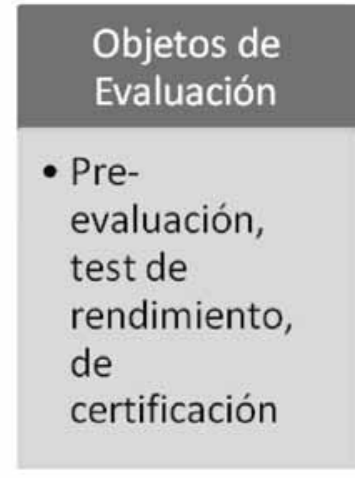

Fig.2 Taxonomía elegida

En base al modelo y a la taxonomía presentada se analizaron herramientas de evaluación de $\mathrm{OA}$, teniendo en cuenta diversos criterios y de acuerdo al contexto en el que el mismo será aplicado. Entre las más destacadas se encuentran:

- $\quad$ LORI (Learning Object Review Instrument) la cual presenta un formulario en línea que contiene ítems a evaluar, escala de valoración y un espacio para los comentarios; y

- HEODAR (Herramienta de Evaluación de Objetos Didácticos de Aprendizaje Reutilizable), como característica importante se integra como un módulo a la plataforma Moodle. Toma como base dos aspectos para su evaluación: el pedagógico, presenta una subdivisión entre lo que se considera psicopedagógico y didáctico-curricular; y el tecnológico tiene en cuenta el diseño de la interfaz y la navegabilidad.

- MERLOT: es un repositorio que permite evaluar los OA que almacena, desde tres puntos de vista o aspectos, calidad del contenido, potencial de efectividad y facilidad de uso.

\section{MÉTODOS Y HERRAMIENTAS}

Las herramientas mencionadas requieren que un OA incluya: contenido, actividades y evaluación. Es decir que no aplica al modelo de OA utilizado en la FRRe que responde a la taxonomía elegida por el Grupo de Investigación (ver Fig.2). Debido a lo antes expuesto se propuso un nuevo instrumento [8], el cual se presenta en la Tabla 1.

La herramienta descripta previamente fue aplicada a la evaluación de OA de las asignaturas Algoritmos y Estructuras de Datos y Física 1, disponibles en la plataforma virtual Moodle. En ambas asignaturas se han evaluado tres objetos de cada tipo, seleccionando cuatro de ellos por similitud en el cumplimiento o no de los aspectos considerados en la evaluación, los mismos son mostrados en la Tabla 2: 


\begin{tabular}{|c|c|c|c|c|}
\hline Aspecto a considerar & $\begin{array}{c}\text { Objeto de } \\
\text { Instrucción }\end{array}$ & $\begin{array}{c}\text { Objeto de } \\
\text { Colaboración }\end{array}$ & $\begin{array}{c}\text { Objeto de } \\
\text { Práctica }\end{array}$ & $\begin{array}{c}\text { Objeto de } \\
\text { Evaluación }\end{array}$ \\
\hline $\begin{array}{l}\text { Objetivos del aprendizaje: queda reflejado } \\
\text { claramente lo que se espera sea aprendido/aplicado }\end{array}$ & Necesario & Necesario & Necesario & Necesario \\
\hline \multicolumn{5}{|l|}{ Contenidos } \\
\hline Coherentes con los objetivos propuestos & Necesario & Necesario & Necesario & Necesario \\
\hline $\begin{array}{l}\text { La información presentada se inicia teniendo como } \\
\text { base los saberes previos del alumno }\end{array}$ & Necesario & Deseado & NO se aplica & NO se aplica \\
\hline El lenguaje es claro y adecuado al nivel del alumno & Necesario & Necesario & Necesario & Necesario \\
\hline $\begin{array}{l}\text { Ejemplificación como medio para aclarar } \\
\text { conceptos }\end{array}$ & Necesario & Deseado & NO se aplica & NO se aplica \\
\hline $\begin{array}{l}\text { La fuente de información es confiable y acorde a la } \\
\text { temática }\end{array}$ & Necesario & Necesario & Deseado & NO se aplica \\
\hline \multicolumn{5}{|l|}{ Actividades } \\
\hline Están orientadas al cumplimiento de los objetivos & NO se aplica & Necesario & Necesario & Necesario \\
\hline $\begin{array}{l}\text { Promueven el desarrollo e iniciativa y el } \\
\text { aprendizaje autónomo }\end{array}$ & NO se aplica & Necesario & Deseado & Deseado \\
\hline Retroalimentación & NO se aplica & Necesario & Necesario & Necesario \\
\hline \multicolumn{5}{|l|}{ Tecnológico } \\
\hline Es accesible & Deseado & Deseado & Deseado & Deseado \\
\hline Usabilidad & Necesario & Necesario & Necesario & Necesario \\
\hline Reusabilidad & Necesario & Deseado & Necesario & Necesario \\
\hline Cumplimento de estándares & Necesario & Necesario & Necesario & Necesario \\
\hline
\end{tabular}

Tabla 1: Aspectos a considerar para la evaluación de objetos de aprendizaje - Aplicación a cada uno de los tipos de objetos presentado en la taxonomía

\begin{tabular}{|c|c|c|c|c|}
\hline Aspecto a considerar & $\begin{array}{c}\text { Objeto de } \\
\text { Instrucción }\end{array}$ & $\begin{array}{c}\text { Objeto de } \\
\text { Colaboración }\end{array}$ & $\begin{array}{l}\text { Objeto de } \\
\text { Práctica }\end{array}$ & $\begin{array}{l}\text { Objeto de } \\
\text { Evaluación }\end{array}$ \\
\hline $\begin{array}{l}\text { Objetivos del aprendizaje: queda reflejado } \\
\text { claramente lo que se espera sea aprendido/aplicado }\end{array}$ & No cumple & Cumple & No cumple & No cumple \\
\hline \multicolumn{5}{|l|}{ Contenidos } \\
\hline Coherentes con los objetivos propuestos & No cumple & Cumple & No cumple & No cumple \\
\hline $\begin{array}{l}\text { La información presentada se inicia teniendo como } \\
\text { base los saberes previos del alumno }\end{array}$ & Cumple & Cumple & - & $\cdot$ \\
\hline El lenguaje es claro y adecuado al nivel del alumno & Cumple & Cumple & Cumple & Cumple \\
\hline $\begin{array}{l}\text { Ejemplificación como medio para aclarar } \\
\text { conceptos }\end{array}$ & Cumple & No cumple & - & $\cdot$ \\
\hline $\begin{array}{l}\text { La fuente de información es confiable y acorde a la } \\
\text { temática }\end{array}$ & No cumple & Cumple & No cumple & $\cdot$ \\
\hline \multicolumn{5}{|l|}{ Actividades } \\
\hline Están orientadas al cumplimiento de los objetivos & - & Cumple & No cumple & No cumple \\
\hline $\begin{array}{l}\text { Promueven el desarrollo e iniciativa y el } \\
\text { aprendizaje autónomo }\end{array}$ & $\cdot$ & Cumple & Cumple & Cumple \\
\hline Retroalimentación & - & Cumple & Cumple & No cumple \\
\hline \multicolumn{5}{|l|}{ Tecnológico } \\
\hline Es accesible & No cumple & No cumple & No cumple & No cumple \\
\hline Usabilidad & Cumple & Cumple & Cumple & No cumple \\
\hline Reusabilidad & Cumple & Cumple & Cumple & Cumple \\
\hline Cumplimento de estándares & No cumple & No cumple & No cumple & No cumple \\
\hline
\end{tabular}

Tabla 2: Evaluación de OA disponibles en la plataforma virtual Moodle 


\section{CONCLUSIONES}

El desarrollo de la propuesta para evaluar los OA responde al modelo y taxonomía adoptada en la FRRe: El instrumento especifica las características que se consideran $\mathrm{Ne}$ cesarias (obligatorias) y Deseadas (no obligatorias) como así también una ponderación que permite interpretar de mejor manera la calidad del OA evaluado.

Respecto de la evaluación, es posible verificar que los $\mathrm{OA}$ analizados no cumplen los criterios de inclusión de los objetivos de aprendizaje, que permite evaluar la coherencia de los contenidos con los objetivos del OA, ni el de inclusión de fuentes de información (o referencias bibliográficas), ambas Necesarias para dichos Objetos. Únicamente cumple con estos criterios el Objeto de colaboración de Física 1.

En el caso del Objeto de práctica es importante destacar que las dos características más importantes de este tipo de $\mathrm{OA}$ se cumplen: la retroalimentación y la promoción del desarrollo, la iniciativa y el aprendizaje autónomo.

También se verifica que el Objeto de evaluación cumple con dos características necesarias y fundamentales para el mismo: la promoción del desarrollo, la iniciativa y el aprendizaje autónomo y la reusabilidad.

El Objeto de colaboración es el que más características Necesarias cumple.

En lo referido al incumplimiento de la característica Retroalimentación en los Objetos de práctica y evaluación, la misma no es automática ya que se trata de tareas asíncronas, en que esa condición es cumplida por el docente al corregir e informar sobre la corrección, por lo que no se podría considerar inexistente.

En cuanto a la Accesibilidad, es importante destacar que los OA alojados en la plataforma analizada no cumplen con dicha condición debido a que la versión instalada, Moodle 1.9 , no implementa este estándar.

Como acciones futuras se pretende concluir la evaluación de los OA de ambas asignaturas a los efectos de realizar una retroalimentación completa a la cátedra. También se prevé seleccionar, al azar, OA de otras asignaturas a efectos de verificar si las coincidencias detectadas en los OA de las dos materias evaluadas se mantienen en las restantes y si se incorporan otras. En función de este estudio será posible proponer capacitaciones dirigidas a los docentes para el mejoramiento del material de enseñanza y aprendizaje, con vistas al desarrollo de un repositorio de objeto de aprendizaje de calidad.

\section{Referencias}

[1] LOPEZ GUZMAN, Clara. (2005) "Los repositorios de OA como soporte para los entornos de e-learning". Tesis doctoral. Universidad de Salamanca. España.

[2] GARCÍA ARETIO (2005). “Objetos de Aprendizaje”. Boletín Electrónico de Noticias de Educación a Distancia - Bened. Disponible en http://e-spacio.uned.es/fez/eserv.php?pid=bibliuned:329\&dsID=editorialfebrero2005.pdf

[3] MASON, R., WELLER, M., \& PEGLER, C. (2003). Learning in the Connected Economy. Londres: Open 


\section{University}

[4] Wiley, D. A. (2000). "Connecting learning objects to instructional design theory: A definition, a metaphor, and a taxonomy”. En D. A. Wiley (2000), The Instructional Use of Learning Objects. Disponible en http://reusability.org/ $\mathrm{read} / \mathrm{chapters} /$ wiley.doc

[5] LOWERISON G.; GALLANT, G. \& BOYD, G. (2003). Learning Objects in Distance Education: Addressing issues of Quality, Learner Control and Accessibility. En Proceedings of the 2003 CADE-ACED Conference. Disponible en http://www.cade-aced2003.ca/conference_proceedings/Gallant.pdf

[6] Álvarez Rodríguez, F.J.; Muñoz Arteaga, J. “Fundamentos del enfoque de objetos de aprendizaje”. Cap.I, pp 20 a 29. Ed. Universidad de AGuas Calientes. Libro electrónico disponible en: http://ingsw.ccbas.uaa.mx/sitio/images/ libro/

[7] ASTD y SmartForce. (2003, sep. 30). A field guide to learning object. Retrivied. Chan Núñez, M.E. (2002). Hacia la construcción de la sociedad de aprendizaje. Apertura. 